\title{
The Uncertainty of Noise Composed of Separate Sound Events
}

\author{
Rufin MAKAREWICZ, Roman GOEĘBIEWSKI \\ Institute of Acoustics, Faculty of Physics, Adam Mickiewicz University \\ Umultowska 85, 61-614 Poznań, Poland; e-mail: roman_g@amu.edu.pl \\ (received January 24, 2015; accepted November 10, 2015)
}

\begin{abstract}
Transportation noise is a main source of noise pollution. It is assumed that it consists of recognizable noise events which come from moving aircrafts, trains and boats. The noise of an isolated sound event is assessed by the sound exposure level, $L_{A E}$. Much legislation and many regulations and guidelines employ the A-weighted time-average sound level, $L_{A e q, T}$, with the time interval Tof one hour or longer. $L_{A E}$ measurements enable an approximation of $L_{A e q, T}$. The key point is the uncertainty of this approximation. It has been shown that an increase in the number of $L_{A E}$ categories brings about a decrease in uncertainty. For illustrative purposes, $L_{A E}$ measurements of aircrafts taking off and landing were carried out.
\end{abstract}

Keywords: noise propagation.

\section{Introduction}

Noise pollution due to transportation (e.g. aircraft noise) has increased over recent years (BABISCH, 2007; Murphy, 2009; Pirrera, 2010). To quantify noise, much legislation and many regulations and guidelines employ the A-weighted time-average sound level, $L_{A e q, T}$, with the time interval $T$ of one hour or longer (Survey, 2009; ANSI, 1960; ISO, 2003). Sometimes, transportation noise is composed of discrete sound events, such as aircraft operations train- and boatpass-bys (Fig. 1). The noise of an isolated sound event is measured in terms of the sound exposure level, $L_{A E}$. Due to differences in noise generation and propagation the measured values of $L_{A E}$ are not identical, so their categorization is possible. The grouping of aircraft operations into take offs and landings is the simplest example (Sec. 3).

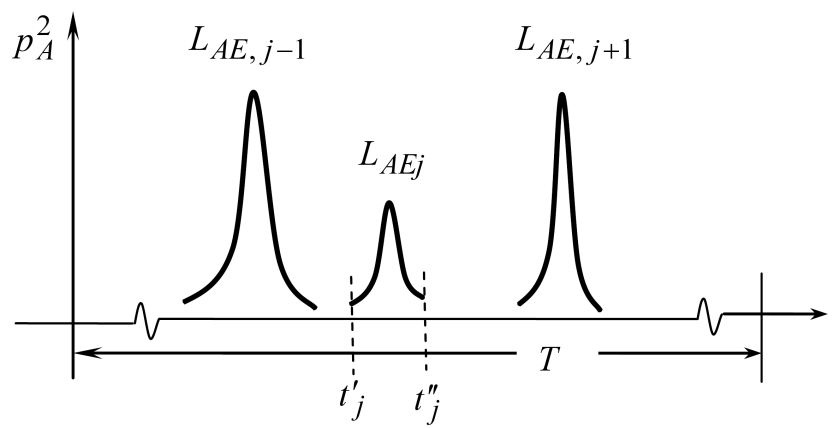

Fig. 1. Environmental noise composed of noise events: $\ldots, L_{A E, j-1}, L_{A E j}, L_{A E, j+1}, \ldots$
This study shows that grouping all $L_{A E}$ into two, three or more categories (Sec. 4) decreases the uncertainty of $L_{A e q, T}$ approximation. For one category of sound events (Sec. 2), this uncertainty can be calculated from (see CAliguri, 2007; Kephalopoulos, 2007; MAKAREWICZ, 2008 and the literature cited therein),

$$
\sigma_{L}=\frac{10}{\ln 10} \frac{\sigma_{\varepsilon}}{\sqrt{q} \cdot\langle\varepsilon\rangle}
$$

Here $q$ expresses the number of $L_{A E}$ measurements. The empirical mean $\langle\varepsilon\rangle$, and the variance, $\sigma_{\varepsilon}^{2}$, of the event sound exposure can be obtained from,

$$
\begin{gathered}
\langle\varepsilon\rangle=\frac{1}{q} \sum_{j=1}^{q} 10^{0.1 L_{A E j}}, \quad\left\langle\varepsilon^{2}\right\rangle=\frac{1}{q} \sum_{j=1}^{q} 10^{0.2 L_{A E j}}, \\
\sigma_{\varepsilon}^{2}=\left\langle\varepsilon^{2}\right\rangle-\langle\varepsilon\rangle^{2} .
\end{gathered}
$$

In Secs. 3 and 4 the generalization of Eq. (1), for two- and more categories, is derived. The new uncertainty is less than $\sigma_{L}$ (Eq. (1)). This is an important result, because any decrease in the uncertainty enhances the reliability of $L_{A e q, T}$ approximation. For illustrative purposes, aircraft noise measurements were carried out. Nevertheless, the results of this study can be used for any type of noise which is composed of recognizable noise events (Fig. 1). 


\section{One category of noise events}

Taking into account $Q$ noise events within the time interval $T$, the combination of definitions of $L_{A e q, T}$ and $L_{A E}$ yields the exact value of the Aweighted time-average sound level (ANSI, 1960; IS0, 2003; MAKAREWICZ, 2008)

$L_{A e q, T}=10 \log \left\{\frac{t_{o}}{T} \sum_{j=1}^{Q} 10^{0.1 L_{A E, j}}\right\}, \quad t_{o}=1 \mathrm{~s}$,

where

$$
L_{A E j}=10 \log \left\{\varepsilon_{j}\right\},
$$

is the $j$-th sound exposure level. With the time pattern of the A-weighted squared sound pressure for the $j$-th event, $p_{A j}^{2}(t)$ (Fig. 1), the integral

$$
\varepsilon_{j}=\int_{t_{j}^{\prime}}^{t_{j}^{\prime \prime}} \frac{p_{A j}^{2}}{p_{o}^{2}} \frac{d t}{t_{o}}, \quad p_{o}=20 \mu \mathrm{Pa},
$$

brings about the event sound exposure. The exact mean of the event sound exposure is,

$$
\bar{\varepsilon}=\frac{1}{Q} \sum_{j=1}^{Q} 10^{0.1 L_{A E j}},
$$

where $Q$ denotes the exact number of noise events within the time interval $T$. Consequently, the exact value of A-weighted time-average sound level (Eq. (3)) can be rewritten as,

$$
L_{A e q, T}=10 \log \left\{\frac{Q t_{o}}{T} \cdot \bar{\varepsilon}\right\},
$$

Suppose that $q<Q$ measurements of the sound exposure level,

$$
L_{A E 1}, \ldots, L_{A E j}, \ldots, L_{A E q},
$$

is representative, i.e. encompasses all possible conditions of sound emission and propagation. Thus the empirical mean of the event sound exposure (Eq. (6)),

$$
\langle\varepsilon\rangle=\frac{1}{q} \sum_{j=1}^{q} 10^{0.1 L_{A E j}},
$$

yields the empirical and representative value of the Aweighted time-average sound level (Eq. (7)),

$$
\widehat{L}_{A e q, T}=10 \log \left\{\frac{Q t_{o}}{T}\langle\varepsilon\rangle\right\} .
$$

Note that the variations in noise generation and propagation could lead to different samples of $L_{A E}$ (Eq. (8)). For such virtual samples one gets virtual means (Eq. (9)), $\langle\varepsilon\rangle_{1}, \ldots,\langle\varepsilon\rangle_{i}, \ldots,\langle\varepsilon\rangle_{n}$, and then virtual A-weighted time-average sound levels (Eq. (10)),

$$
L_{\text {Aeq }, T}^{(1)}, \ldots, L_{\text {Aeq }, T}^{(i)}, \ldots, L_{\text {Aeq }, T}^{(n)},
$$

which are calculated from,

$$
L_{\text {Aeq,T }}^{(i)}=10 \log \left\{\frac{Q t_{o}}{T}\langle\varepsilon\rangle_{i}\right\}, \quad i=1, \ldots, n .
$$

Note that the number of noise events, $Q$, within the time interval $T$, remains constant. Now, introducing the identity, $\langle\varepsilon\rangle_{i}=\langle\varepsilon\rangle+\langle\varepsilon\rangle_{i}-\langle\varepsilon\rangle$, with the empirical mean $\langle\varepsilon\rangle$ (Eq. (9)), and applying approximation, $\log (1+x) \approx x / \ln (10)$, we arrive at (Eqs. (10), (12)),

$$
L_{A e q, T}^{(i)} \approx \widehat{L}_{A e q, T}+\frac{10}{\ln (10)} \frac{\langle\varepsilon\rangle_{i}-\langle\varepsilon\rangle}{\langle\varepsilon\rangle} .
$$

Consequently, Eqs. (11) and (13) imply that the mean of the virtual A-weighted time-average sound levels, $L_{\text {Aeq,T }}^{(i)}$, comes near to the empirical A-weighted timeaverage sound level,

$$
\frac{1}{n} \sum_{i=1}^{n} L_{A e q, T}^{(i)} \approx \widehat{L}_{A e q, T} .
$$

On the other hand, $\widehat{L}_{A e q, T}$ (Eq. (10)) is representative for all possible conditions and approximates the exact A-weighted time-average sound level (Eq. (3)),

$$
L_{A e q, T} \approx \widehat{L}_{A e q, T} .
$$

The uncertainty of the above approximation estimates the standard deviation, $\sigma_{L}$, defined by (Cremer, 1999),

$$
\sigma_{L}^{2}=\frac{1}{n} \sum_{i=1}^{n}\left[L_{\text {Aeq,T }}^{(i)}-\widehat{L}_{A e q, T}\right]^{2}
$$

and the combination of Eqs. (2), (4), (13) and (16) leads to the well known formula (1).

Example 1. $L_{A E}$ measurements of aircraft operations were performed in the vicinity of a one runway airport. To the more instructive we disregard differences between aircraft types, flight profiles, meteorological conditions, etc., and assume one category of noise events. The sample of the measured sound exposure levels,

$$
\begin{aligned}
& L_{A E j}=80.3,65.2,75.1,72.1,78.8,78.7 \text {, } \\
& 86.4, \quad 68.1, \quad 88.2, \quad 69.1, \quad 77.9, \quad 73.2 \text {, } \\
& 84.1,78.6,83.6,69.5 \mathrm{~dB} \text {, }
\end{aligned}
$$

is characterized by (Eq. (2)): $\langle\varepsilon\rangle \approx 12.9 \cdot 10^{7}$ and $\sigma_{\varepsilon}^{2} \approx$ $31.9 \cdot 10^{15}$. For the number of measurements $q=16$, formula (1) yields the uncertainty, $\sigma_{L}=1.5 \mathrm{~dB}$, of the A-weighted time-average sound level approximation (15). 


\section{Two categories of noise events}

This section shows that the splitting of the measurement sample of $q$ elements (Eq. (8)) into two subsamples

$$
\text { and } \begin{aligned}
& L_{A E 1}^{(1)}, \ldots, L_{A E j}^{(1)}, \ldots, L_{A E, q 1}^{(1)}, \\
& L_{A E 1}^{(2)}, \ldots, L_{A E j}^{(2)}, \ldots, L_{A E, q 2}^{(2)},
\end{aligned}
$$

with $q_{1}+q_{2}=q$, decreases standard deviation, $\breve{\sigma}_{L}<$ $\sigma_{L}$. In order to find the new value of $\breve{\sigma}_{L}$, we employ two categories of noise events. For the actual number of noise events, $Q=Q_{1}+Q_{2}$, the exact A-weighted time-average level takes the form (Eqs. (3), (7)),

$$
L_{A e q, T}=10 \log \left\{\frac{t_{o}}{T}\left[Q_{1} \cdot \bar{\varepsilon}_{1}+Q_{2} \cdot \bar{\varepsilon}_{2}\right]\right\},
$$

where the exact means of the event sound exposures are,

$$
\bar{\varepsilon}_{1}=\frac{1}{Q_{1}} \sum_{j=1}^{Q_{1}} \varepsilon_{1 j}, \quad \bar{\varepsilon}_{2}=\frac{1}{Q_{2}} \sum_{j=1}^{Q_{2}} \varepsilon_{2 j} .
$$

To determine estimations, $\left\langle\varepsilon_{1}\right\rangle \approx \bar{\varepsilon}_{1}$, and $\left\langle\varepsilon_{2}\right\rangle \approx \bar{\varepsilon}_{2}$, the measurements of $L_{A E}^{(1)}$ and $L_{A E}^{(2)}$ for both categories (Eq. (17)), have to be performed. The empirical means, $\left\langle\varepsilon_{1}\right\rangle ;\left\langle\varepsilon_{2}\right\rangle$, second moments, $\left\langle\varepsilon_{1}^{2}\right\rangle ;\left\langle\varepsilon_{2}^{2}\right\rangle$, and variances, $\sigma_{\varepsilon 1}^{2} ; \sigma_{\varepsilon 2}^{2}$, are obtained from Eq. (2), with $q$ replaced by $q_{1}$ and $q_{2}$, respectively.

Example 2. In the vicinity of the one runway airport (see Example 1), $L_{A E}$ measurements of noise produced during take off and landing were carried out:

$$
\begin{aligned}
& L_{A E j}^{(1)}=80.3,75.1,78.8,86.4,88.2, \\
& 77.9,84.1,83.6 \mathrm{~dB} \text {, } \\
& L_{A E j}^{(2)}=65.2, \quad 72.1, \quad 78.7,68.1,69.1 \text {, } \\
& 73.2,78.6,69.5 \mathrm{~dB} \text {. }
\end{aligned}
$$

With $q_{1}=q_{2}=8$ the characteristics of $\varepsilon_{1}$ and $\varepsilon_{2}$, are as follows (Eq. (2)):

$$
\begin{array}{rlrl}
\left\langle\varepsilon_{1}\right\rangle & \approx 23.3 \cdot 10^{7} ; \quad \sigma_{\varepsilon 1}^{2} & \approx 41.9 \cdot 10^{15}, \\
\text { and } \quad\left\langle\varepsilon_{2}\right\rangle & \approx 2.63 \cdot 10^{7} ; \quad \sigma_{\varepsilon 1}^{2} \approx 76.3 \cdot 10^{13} .
\end{array}
$$

The approximation of the exact A-weighted time average sound level for two categories of sound events is, $L_{A e q, T} \approx \breve{L}_{A e q, T}$ (compare with Eq. (15)), where

$$
\breve{L}_{A e q, T}=10 \log \left\{\frac{t_{o}}{T}\left[Q_{1} \cdot\left\langle\varepsilon_{1}\right\rangle+Q_{2} \cdot\left\langle\varepsilon_{2}\right\rangle\right]\right\} .
$$

Here the empirical means, $\left\langle\varepsilon_{1}\right\rangle$ and $\left\langle\varepsilon_{2}\right\rangle$, come from measurements of $L_{A E}^{(1)}$ and $L_{A E}^{(2)}$, respectively
(Eq. (17)). However, variations in noise generation and propagation could lead to different means,

$$
\begin{aligned}
& \left\langle\varepsilon_{1}\right\rangle_{1}, \ldots,\left\langle\varepsilon_{1}\right\rangle_{i}, \ldots,\left\langle\varepsilon_{1}\right\rangle_{n} \\
\text { and } & \left\langle\varepsilon_{2}\right\rangle_{1}, \ldots,\left\langle\varepsilon_{2}\right\rangle_{i}, \ldots,\left\langle\varepsilon_{2}\right\rangle_{n}
\end{aligned}
$$

and different values of the virtual A-weighted time average sound level,

$$
\begin{aligned}
\breve{L}_{\text {Aeq,T }}^{(i)} & =10 \log \left\{\frac{t_{o}}{T}\left[Q_{1} \cdot\left\langle\varepsilon_{1}\right\rangle_{i}+Q_{2} \cdot\left\langle\varepsilon_{2}\right\rangle_{i}\right]\right\}, \\
i & =1, \ldots, n .
\end{aligned}
$$

As in Sec. 2, now we apply the empirical means $\left\langle\varepsilon_{1}\right\rangle ;\left\langle\varepsilon_{2}\right\rangle$ (Eq. (2), Example 2), and write identities for the virtual means of the first and the second category of noise events,

$$
\begin{aligned}
& \left\langle\varepsilon_{1}\right\rangle_{i}=\left\langle\varepsilon_{1}\right\rangle+\left\langle\varepsilon_{1}\right\rangle_{i}-\left\langle\varepsilon_{1}\right\rangle, \\
& \left\langle\varepsilon_{2}\right\rangle_{i}=\left\langle\varepsilon_{2}\right\rangle+\left\langle\varepsilon_{2}\right\rangle_{i}-\left\langle\varepsilon_{2}\right\rangle .
\end{aligned}
$$

Finally, Eqs. (22), (23) and the approximation $\log (1+x) \approx x / \ln (10)$ result in the $i$-th virtual Aweighted time average sound level,

$$
\begin{aligned}
L_{\text {Aeq }, T}^{(i)} \approx & \breve{L}_{A e q, T}+\frac{10}{\ln 10} \\
& \cdot \frac{Q_{1}\left[\left\langle\varepsilon_{1}\right\rangle_{i}-\left\langle\varepsilon_{1}\right\rangle\right]+Q_{2}\left[\left\langle\varepsilon_{2}\right\rangle_{i}-\left\langle\varepsilon_{2}\right\rangle\right]}{Q_{1} \cdot\left\langle\varepsilon_{1}\right\rangle+Q_{2} \cdot\left\langle\varepsilon_{2}\right\rangle} .
\end{aligned}
$$

The level $\breve{L}_{A e q, T}$ (Eq. (20)) expresses the mean of the above levels, $L_{\text {Aeq,T }}^{(i)}$ (Eqs. (14), (24)), and their variance can be found from (compare with Eq. (16)),

$$
\breve{\sigma}_{L}^{2}=\frac{1}{n} \sum_{i=1}^{n}\left[L_{\text {Aeq,T }}^{(i)}-\breve{L}_{A e q, T}\right]^{2} .
$$

With some calculations the above Eqs. (24) and (25) combine into the new variance for two categories of noise events,

$$
\breve{\sigma}_{L}=\frac{10}{\ln 10} \cdot \frac{\sqrt{Q_{1}^{2} \frac{\sigma_{\varepsilon 1}^{2}}{q_{1}}+Q_{2}^{2} \frac{\sigma_{\varepsilon 2}^{2}}{q_{2}}}}{Q_{1}\left\langle\varepsilon_{1}\right\rangle+Q_{2}\left\langle\varepsilon_{2}\right\rangle} .
$$

For the same number of noise events in both categories, $Q_{1}=Q_{2}=Q / 2$, and the same number of $L_{A E}$ measurements, $q_{1}=q_{2}=q / 2$, the above relationship translates into,

$$
\breve{\sigma}_{L}=\frac{10}{\ln 10} \frac{\sqrt{2\left(\sigma_{\varepsilon 1}^{2}+\sigma_{\varepsilon 2}^{2}\right)}}{\sqrt{q}\left[\left\langle\varepsilon_{1}\right\rangle+\left\langle\varepsilon_{2}\right\rangle\right]} .
$$

If the differences between two categories of noise events disappear (e.g. $L_{A E}$ measurements of train noise far away from the double-track railway), then $\sigma_{\varepsilon 1} \approx$ $\sigma_{\varepsilon 2}$ and $\left\langle\varepsilon_{1}\right\rangle \approx\left\langle\varepsilon_{2}\right\rangle$. Ultimately, Eqs. (1) and (27) yield identical standard deviations, $\sigma_{L}=\breve{\sigma}_{L}$, as expected. 
Example 3. In Example 2 we made use of $q_{1}=$ $q / 2=8$ values of $L_{A E}^{(1)}$ (take off) and $q_{2}=q / 2=8$ values of $L_{A E}^{(2)}$ (landing). Substitution of $q=16,\left\langle\varepsilon_{1}\right\rangle \approx$ $23.3 \cdot 10^{7} ; \sigma_{\varepsilon 1}^{2} \approx 41.9 \cdot 10^{15}$, and $\left\langle\varepsilon_{2}\right\rangle \approx 2.63 \cdot 10^{7}$; $\sigma_{\varepsilon 1}^{2} \approx 76.3 \cdot 10^{13}$ into Eq. (27) gives the new standard deviation, $\breve{\sigma}_{L}=1.2 \mathrm{~dB}$, for two categories of sound events. In Example 2 the classic standard deviation, $\sigma_{L}=1.5 \mathrm{~dB}$, for a single category of noise event was calculated.

Example 4. The aircraft noise measurements described in Example 2 were repeated 5 more times. The number of measurements were: $q_{1}=q / 2=5,7,5$, 6,6 (take off operations) and $q_{2}=q / 2=5,7,5,6,6$ (landing operations). The corresponding values of $\left\langle\varepsilon_{1}\right\rangle$; $\sigma_{\varepsilon 1}^{2}$ and $\left\langle\varepsilon_{2}\right\rangle ; \sigma_{\varepsilon 2}^{2}$ are displayed in the first row of Table 1 . The first and the second rows of Table 2 contain $\sigma_{L}$ (classic standard deviation for one category sound events - Eq. (1)) and $\breve{\sigma}_{L}$ (the new standard deviation for two categories of sound events, Eq. (27)). Note that in each case, $\breve{\sigma}_{L}<\sigma_{L}$ (Table 2).

Table 1. The empirical means, $\langle\varepsilon\rangle,\left\langle\varepsilon_{1}\right\rangle ;\left\langle\varepsilon_{2}\right\rangle$ and variances $\sigma_{\varepsilon}^{2}, \sigma_{\varepsilon 1}^{2} ; \sigma_{\varepsilon 2}^{2}$ (Eq. (2)).

\begin{tabular}{|c|c|c|c|c|c|c|}
\hline$q / 2$ & 8 & 5 & 7 & 5 & 6 & 6 \\
\hline$\langle\varepsilon\rangle[\mathrm{E}+07]$ & 12.9 & 5.59 & 13.5 & 4.73 & 17.7 & 16.4 \\
\hline$\sigma_{\varepsilon}^{2}[\mathrm{E}+15]$ & 31.9 & 2.77 & 37.1 & 0.89 & 56.1 & 84.9 \\
\hline$\left\langle\varepsilon_{1}\right\rangle[\mathrm{E}+07]$ & 23.3 & 9.68 & 31.1 & 7.23 & 37.4 & 50.2 \\
\hline$\sigma_{\varepsilon 1}^{2}[\mathrm{E}+15]$ & 41.9 & 1.21 & 40.9 & 0.29 & 68.3 & 110 \\
\hline$\left\langle\varepsilon_{2}\right\rangle[\mathrm{E}+07]$ & 2.63 & 0.45 & 1.75 & 2.22 & 3.69 & 1.38 \\
\hline$\sigma_{\varepsilon 2}^{2}[\mathrm{E}+13]$ & 76.3 & 1.55 & 8.38 & 22.8 & 25.5 & 24.1 \\
\hline
\end{tabular}

Table 2. Example 4: standard deviations for a single category of noise events, $\sigma_{L}$ (Eq. (1)), and for two categories of noise events, $\breve{\sigma}_{L}$ (Eq. (27)).

\begin{tabular}{|c|c|c|c|c|c|c|}
\hline & 1 & 2 & 3 & 4 & 5 & 6 \\
\hline$\sigma_{L}[\mathrm{~dB}]$ & 1.5 & 1.3 & 1.7 & 0.9 & 1.7 & 2.2 \\
\hline$\widehat{\sigma}_{L}[\mathrm{~dB}]$ & 1.2 & 0.7 & 0.7 & 0.2 & 1.1 & 1.1 \\
\hline
\end{tabular}

\section{Many categories of noise events}

From the measured sound exposure levels of noise events which pertain to the $i$-th category

$$
L_{A E 1}^{(i)}, \ldots, L_{A E j}^{(k)}, \ldots,
$$

one gets the empirical mean, second moment, and variance of the event sound exposures (Eq. (2)),

$$
\begin{aligned}
\left\langle\varepsilon_{k}\right\rangle & =\frac{1}{q_{k}} \sum_{j=1}^{q_{i}} 10^{0.1 L_{A E j}^{(k)},} \\
\left\langle\varepsilon_{k}^{2}\right\rangle & =\frac{1}{q_{k}} \sum_{j=1}^{q_{i}} 10^{0.2 L_{A E j}^{(k)}}, \\
\sigma_{\varepsilon k}^{2} & =\left\langle\varepsilon_{k}^{2}\right\rangle-\left\langle\varepsilon_{k}\right\rangle^{2} .
\end{aligned}
$$

The exact A-weighted time average sound level, $L_{A e q, T}$ (Eq. (3)), is approximated by the empirical A-weighted time average sound level,

$$
L_{A e q, T}^{*}=10 \log \left\{\frac{t_{o}}{T}\left[Q_{1}\left\langle\varepsilon_{1}\right\rangle+\ldots+Q_{k}\left\langle\varepsilon_{k}\right\rangle+\ldots\right]\right\},
$$

where $Q_{k}$ expresses the exact number of noise events of the $i$-th category within the time interval $T$. The uncertainty of the approximation, $L_{A e q, T} \approx L_{A e q, T}^{*}$ (Eqs. (3), (30)), can be obtained from the generalization of Eq. (26),

$$
\sigma_{L}^{*}=\frac{10}{\ln 10} \cdot \frac{\sqrt{Q_{1}^{2} \frac{\sigma_{\varepsilon 1}^{2}}{q_{1}}+\ldots+Q_{k}^{2} \frac{\sigma_{\varepsilon k}^{2}}{q_{k}}+\ldots}}{Q_{1}\left\langle\varepsilon_{1}\right\rangle+\ldots+Q_{k}\left\langle\varepsilon_{k}\right\rangle+\ldots} .
$$

If all noise events belong to a single category, $Q_{1} \rightarrow$ $Q=Q_{1}+\ldots+Q_{k}+\ldots, q_{1} \rightarrow q=q_{1}+\ldots+q_{k}+\ldots$, and $Q_{2}=0, \ldots, Q_{k}=0$, then Eq. (30) and (31) describe the case of one category of sound events, which is quantified by Eqs. (1), (2) and (7), (10).

\section{Conclusions}

Equation (31) is the key result of this study. Without categorization of noise events, the a priori knowledge of the number of noise events $Q$ (within the time interval $T$ ), and $q$ measurements of the sound exposure level, $L_{A E}$ (Eq. (8)), enables the approximation of the A-weighted time-average sound level as follows (Eqs. (2), (3), (10)):

$$
L_{A e q, T} \approx 10 \log \left\{\frac{Q t_{o}}{T}\langle\varepsilon\rangle\right\}
$$

The well-known relationship given by Eq. (1) defines its uncertainty, $\sigma_{L}$. A few noise event categories, with $q_{1}$ measurements of $L_{A E}^{(1)}, q_{2}$ measurements of $L_{A E}^{(2)}, \ldots$, etc., leads to the approximation (Eqs. (2), (3), (30)):

$$
L_{A e q, T} \approx 10 \log \left\{\frac{t_{o}}{T}\left[Q_{1}\left\langle\varepsilon_{1}\right\rangle+Q_{2}\left\langle\varepsilon_{2}\right\rangle+\ldots\right]\right\},
$$

with the uncertainty $\sigma_{L}^{*}$ (Eq. (31)). Here the actual number of noise events $-Q$, and the number of $L_{A E}$ measurements $-q$ meet the conditions: $Q_{1}+Q_{2}+\ldots=$ $Q$ and $q_{1}+q_{2}+\ldots=q$. The approximation (33) is more reliable than the approximation (31) due to inequality, $\sigma_{L}^{*}<\sigma_{L}$ (Eqs. (1), (31)). The presented above results are applicable, when nose events are clearly recognizable.

\section{Appendix: Two categories versus one category}

To clarify the difference between uncertainties for one sound event category and two sound events categories (Eqs. (1), (26)), first we apply the classis formula (1) for the sample of event sound exposures, 
$\varepsilon_{1}, \ldots, \varepsilon_{q}$. Then, calculating $\langle\varepsilon\rangle,\left\langle\varepsilon^{2}\right\rangle$ and $\sigma_{\varepsilon}^{2}$ we take into account that this sample consists of two subsamples: $q / 2$ elements belong to the first category and the remaining $q / 2$ elements to the second category of noise events. Accordingly, we get the mean and the second moment of $\varepsilon$ :

$$
\begin{aligned}
\langle\varepsilon\rangle & =\frac{1}{q} \sum_{j=1}^{q} \varepsilon_{j}=\frac{1}{q}\left[\sum_{j=1}^{q / 2} \varepsilon_{1 j}+\sum_{j=1}^{q / 2} \varepsilon_{2 j}\right] \\
& =\frac{1}{2}\left[\left\langle\varepsilon_{1}\right\rangle+\left\langle\varepsilon_{2}\right\rangle\right], \\
\left\langle\varepsilon^{2}\right\rangle & =\frac{1}{q} \sum_{j=1}^{q} \varepsilon_{j}^{2}=\frac{1}{q}\left[\sum_{j=1}^{q / 2} \varepsilon_{1 j}^{2}+\sum_{j=1}^{q / 2} \varepsilon_{2 j}^{2}\right] \\
& =\frac{1}{2}\left[\left\langle\varepsilon_{1}^{2}\right\rangle+\left\langle\varepsilon_{2}^{2}\right\rangle\right] .
\end{aligned}
$$

Then variance, $\sigma_{\varepsilon}^{2}=\left\langle\varepsilon^{2}\right\rangle-\langle\varepsilon\rangle^{2}$, takes the form,

$$
\sigma_{\varepsilon}^{2}=\frac{1}{2}\left[\sigma_{\varepsilon 1}^{2}+\sigma_{\varepsilon 2}^{2}\right]+\frac{1}{4}\left[\left\langle\varepsilon_{1}\right\rangle-\left\langle\varepsilon_{2}\right\rangle\right]^{2}
$$

and combination with classic equation (1) brings about the uncertainty of $L_{A e q, T}$ approximation,

$$
\begin{aligned}
\sigma_{L}^{2}= & \left(\frac{10}{\ln 10}\right)^{2} \frac{1}{q}\left\{2 \cdot \frac{\sigma_{\varepsilon 1}^{2}+\sigma_{\varepsilon 2}^{2}}{\left[\left\langle\varepsilon_{1}\right\rangle+\left\langle\varepsilon_{2}\right\rangle\right]^{2}}\right. \\
& \left.+\left[\frac{\left\langle\varepsilon_{1}\right\rangle-\left\langle\varepsilon_{2}\right\rangle}{\left\langle\varepsilon_{1}\right\rangle+\left\langle\varepsilon_{2}\right\rangle}\right]^{2}\right\} .
\end{aligned}
$$

Note that $\sigma_{L}$ is expressed in terms of $\sigma_{\varepsilon 1} ; \sigma_{\varepsilon 2} ;\left\langle\varepsilon_{1}\right\rangle$; $\left\langle\varepsilon_{2}\right\rangle$, though the summation in Eqs. (35) and (36) runs over $q$ elements, as it would be only one category of noise events. On the other hand, the variance $\breve{\sigma}_{L}$, for two noise event categories and the same number of events and measurements $\left(Q_{1}=Q_{2}=Q / 2, q_{1}=q_{2}=\right.$ $q / 2$ ), is given by Eq. (28). Mindful of Eqs. (37) and (28) one arrives at the ratio of the classic and new variances,

$$
\frac{\sigma_{L}^{2}}{\breve{\sigma}_{L}^{2}}=1+\frac{1}{2} \frac{\left[\left\langle\varepsilon_{1}\right\rangle-\left\langle\varepsilon_{2}\right\rangle\right]^{2}}{\sigma_{\varepsilon 1}^{2}+\sigma_{\varepsilon 2}^{2}} .
$$

To grasp the meaning of this relation, suppose $\varepsilon_{1}$ and $\varepsilon_{2}$ are distributed with large variances, $\sigma_{\varepsilon 1}^{2} ; \sigma_{\varepsilon 2}^{2}$, and empirical means close to each other, $\left\langle\varepsilon_{1}\right\rangle \rightarrow\left\langle\varepsilon_{2}\right\rangle$ (Fig. 2),

$$
\left[\left\langle\varepsilon_{1}\right\rangle-\left\langle\varepsilon_{2}\right\rangle\right]^{2} \ll 2 \cdot\left[\sigma_{\varepsilon 1}^{2}+\sigma_{\varepsilon 2}^{2}\right] .
$$

Under such conditions, Eq. (38) implies that splitting sound events into two categories does not significantly decrease the uncertainty of $L_{A e q, T}$ approximation: $\breve{\sigma}_{L} \approx \sigma_{L}$. Conversely, if the categories are clearly

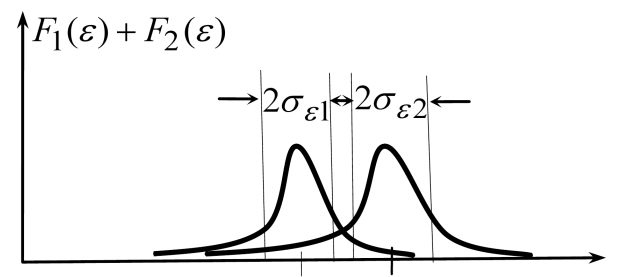

$\left\langle\varepsilon_{1}\right\rangle\left\langle\varepsilon_{2}\right\rangle$

Fig. 2. Probability density function of the event sound exposure, $F_{1}(\varepsilon)+F_{2}(\varepsilon)$, for large variances, $\sigma_{\varepsilon 1}^{2} ; \sigma_{\varepsilon 2}^{2}$, and empirical means close to each other, $\left\langle\varepsilon_{1}\right\rangle \approx\left\langle\varepsilon_{2}\right\rangle$ (Eq. (39)).

separated, $\left\langle\varepsilon_{2}\right\rangle>\left\langle\varepsilon_{1}\right\rangle$ or $\left\langle\varepsilon_{2}\right\rangle<\left\langle\varepsilon_{1}\right\rangle$, and their variances $\sigma_{\varepsilon 1}^{2}$ and $\sigma_{\varepsilon 2}^{2}$ are small (Fig. 3):

$$
\left[\left\langle\varepsilon_{1}\right\rangle-\left\langle\varepsilon_{2}\right\rangle\right]^{2}>2 \cdot\left[\sigma_{\varepsilon 1}^{2}+\sigma_{\varepsilon 2}^{2}\right]
$$

then splitting noise events into two categories decreases the uncertainty of $L_{A e q, T}$ approximation, $\breve{\sigma}_{L}<\sigma_{L}$.

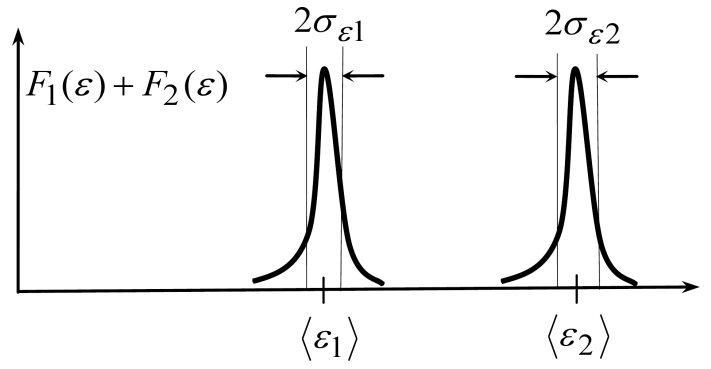

Fig. 3. Probability density function of the event sound exposure, $F_{1}(\varepsilon)+F_{2}(\varepsilon)$, for small variances, $\sigma_{\varepsilon 1}^{2} ; \sigma_{\varepsilon 2}^{2}$, and empirical means clearly separated, $\left\langle\varepsilon_{1}\right\rangle\left\langle\left\langle\varepsilon_{2}\right\rangle\right.$ (Eq. (40)).

Now we reject the assumptions of there being the same number of sound events within the time interval $T, Q_{1}=Q_{2}=Q / 2$, and the same number of measurements, $q_{1}=q_{2}=q / 2$. So the ratios

$$
p=\frac{Q_{1}}{Q}=\frac{q_{1}}{q}, \quad 1-p=\frac{Q_{2}}{Q}=\frac{q_{2}}{q},
$$

express the probabilities that a sound event belongs to the first and the second category, respectively. Here $Q=Q_{1}+Q_{2}$ equals the total number of noise events and $q=q_{1}+q_{2}$ denotes the total number of $L_{A E}$ measurements. Accordingly, for two categories the uncertainty of $L_{A e q, T} \approx \widehat{L}_{A e q, T}$ (Eqs. (3), (20)) estimation can be found from (Eq. (26)),

$$
\breve{\sigma}_{L}^{2}=\left(\frac{10}{\ln 10}\right)^{2} \frac{1}{q} \frac{p \cdot \sigma_{\varepsilon 1}^{2}+(1-p) \cdot \sigma_{\varepsilon 2}^{2}}{\left[p \cdot\left\langle\varepsilon_{1}\right\rangle+(1-p) \cdot\left\langle\varepsilon_{2}\right\rangle\right]^{2}} .
$$

Note that identical probabilities, $p=1 / 2$ and $1-p=$ $1 / 2$ (e.g. the same numbers of take offs and landings) modify the above expression to the form given by Eq. (27).

In order to prove that sound event grouping decreases uncertainty, $\sigma_{L} / \breve{\sigma}_{L}>1$, for any probability, 
$0<p<1$, we have to generalize Eq. (36). The empirical mean and the second moment of the event sound exposure are (Eqs. (2), (4), (41)),

$$
\begin{aligned}
\langle\varepsilon\rangle & =p \cdot\left\langle\varepsilon_{1}\right\rangle+(1-p) \cdot\left\langle\varepsilon_{2}\right\rangle, \\
\left\langle\varepsilon^{2}\right\rangle & =p \cdot\left\langle\varepsilon_{1}^{2}\right\rangle+(1-p) \cdot\left\langle\varepsilon_{2}^{2}\right\rangle
\end{aligned}
$$

and its variance becomes (Eqs. (2), (43)),

$$
\sigma_{\varepsilon}^{2}=p \cdot \sigma_{\varepsilon 1}^{2}+(1-p) \cdot \sigma_{\varepsilon 2}^{2}+p(1-p) \cdot\left[\left\langle\varepsilon_{1}\right\rangle-\left\langle\varepsilon_{2}\right\rangle\right]^{2} .
$$

Inserting the above $\langle\varepsilon\rangle$ and $\sigma_{\varepsilon}^{2}$ into formula (1) one arrives at the generalization of Eq. (36),

$$
\begin{aligned}
\sigma_{L}^{2}= & \left(\frac{10}{\ln 10}\right)^{2} \frac{1}{q}\left[p \cdot\left\langle\varepsilon_{1}\right\rangle+(1-p) \cdot\left\langle\varepsilon_{2}\right\rangle\right. \\
& \left.+p(1-p)\left[\left\langle\varepsilon_{1}\right\rangle-\left\langle\varepsilon_{2}\right\rangle\right]^{2}\right] /\left[\left[p \cdot\left\langle\varepsilon_{1}\right\rangle\right.\right. \\
& \left.\left.+(1-p) \cdot\left\langle\varepsilon_{2}\right\rangle\right]^{2}\right] .
\end{aligned}
$$

Ultimately, combination of formulae (42) and (45) yields the ratio,

$$
\frac{\sigma_{L}^{2}}{\widehat{\sigma}_{L}^{2}}=1+f(p)
$$

The function of the probability, $0<p<1$ (Eq. (43)),

$$
f(p)=\frac{p(1-p) \cdot\left[\left\langle\varepsilon_{1}\right\rangle-\left\langle\varepsilon_{2}\right\rangle\right]^{2}}{p \cdot \sigma_{\varepsilon 1}^{2}+(1-p) \cdot \sigma_{\varepsilon 2}^{2}},
$$

has a maximum at

$$
p=\frac{\sigma_{\varepsilon 2}}{\sigma_{\varepsilon 1}+\sigma_{\varepsilon 2}} .
$$

The corresponding maximum of $\sigma_{L}^{2} / \breve{\sigma}_{L}^{2}$ equals (Eqs. (46), (47)),

$$
\max \left\{\frac{\sigma_{L}^{2}}{\widetilde{\sigma}_{L}^{2}}\right\}=1+\left[\frac{\left\langle\varepsilon_{1}\right\rangle-\left\langle\varepsilon_{2}\right\rangle}{\sigma_{\varepsilon 1}+\sigma_{\varepsilon 2}}\right]^{2} .
$$

Consequently, for two categories of noise events the minimal uncertainty equals,

$$
\widehat{\sigma}_{L}=\frac{\sigma_{L}}{\sqrt{1+\left[\frac{\left\langle\varepsilon_{1}\right\rangle-\left\langle\varepsilon_{2}\right\rangle}{\sigma_{\varepsilon 1}+\sigma_{\varepsilon 2}}\right]^{2}}} .
$$

Here $\sigma_{L}$ (Eq. (1)) represents the uncertainty of estimation, $L_{A e q, T} \approx \widehat{L}_{A e q, T}$ (Eqs. (3), (10)), for noise events belonging to a single category.

\section{References}

1. ANSI S1.1-1960. Acoustical Terminology, 1960. American National Standards Institute, New York.

2. Babisch W., Houthuiss D., Pershagen G., Cadum E., Katsouyanni K., Velonakis M., Dudley M., Marohn H., Swart W., Breugelmans O., Bluhm G., Selander J., Vigna-Taglianti F., Pisani S., Haralabidis A., Dimakopoulou K., ZaChOs I., JÄRUP L., HYENA Consortium (2009), $A n$ noyance due to aircraft noise has increased over the years-result of the HYENA study, Environmental International, 35, 1169-1176.

3. Caligiuri L.M. (2007), The evaluation of uncertainty in environmental acoustic measurements according to the ISO Guide, Noise Control Eng. J., 55, 116-132.

4. Cramer H. (1999), Mathematical Methods of Statistics, Princeton University Press, Princeton.

5. ISO 1996-1. Acoustics - Description, measurement and assessment of environmental noise - Part 1: Basic quantities and assessment procedures, 2003. International Organization for Standardization, Geneva.

6. ISO, Evaluation of measurement data - Guide to expression of uncertainty in measurements, JCGM 100, 2008.

7. Kephalopoulos S., Paviotti K., Knauss D., BERENGIER M. (2007), Uncertainties in long-term road noise monitoring including meteorological influences, Noise Control Eng. J., 55, 133-141.

8. Makarewicz R., ZóŁtowski M. (2008), Variations of road traffic noise in residential areas, J. Acoust. Soc. Am., 24, 3568-3575.

9. Murphy E., King E., Rice H. (2009), Estimation human exposure to transportation noise in central Dublin, Environment International, 35, 298-302.

10. Pirrera S., De Valck E., Cluydts R. (2010), Nocturnal road tarffic noise: A review on its assessment and consequences on sleep and health, Environment International, 36, 492-498.

11. Survey of legislation, regulations, and guidelines for control of community noise, Technical Study Group on Noise Policies and Regulations (TSG 3), July, 2009. 